\title{
Produção de alimentos na base do feijão-caupi (Vigna unguiculata): importância nutricional e benefícios para a saúde
}

\author{
Food production based on cowpea (Vigna unguiculata): nutritional importance and health benefits
}

Producción de alimentos a base de caupí (Vigna unguiculata): importancia nutricional y beneficios

para la salud

Recebido: 13/05/2021 | Revisado: 22/05/2021 | Aceito: 23/10/2021 | Publicado: 24/10/2021

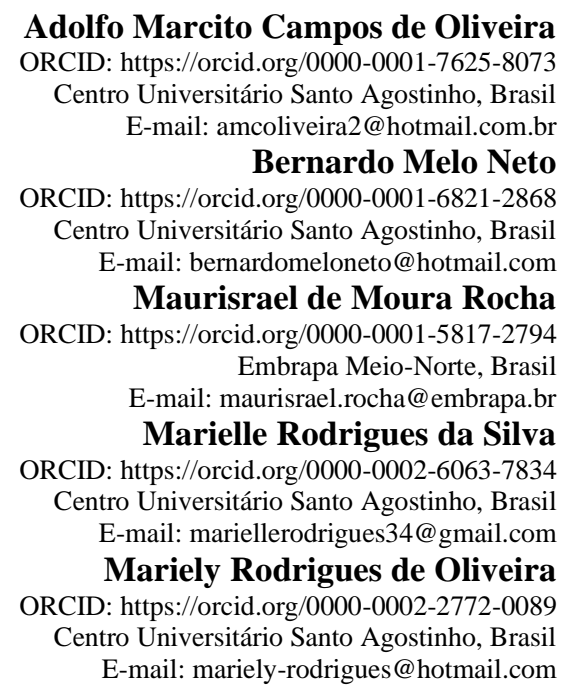

\section{Resumo}

O desenvolvimento e enriquecimento de alimentos têm grande importância não só para a indústria, como também para a melhoria da nutrição da população, onde se pode criar novos produtos ou otimizar os já existentes. O feijão-caupi constitui-se um dos principais componentes da dieta alimentar nas regiões Nordeste e Norte do Brasil, especialmente na zona rural, em virtude do seu alto valor nutritivo, há uma necessidade de ampliar a diversificação de formulações com essa matéria-prima, no intuito de aumentar a oferta de produtos de baixo custo, principalmente para a população de baixa renda. Este trabalho tem como objetivo identificar quais as principais formulações desenvolvidas, contendo como matéria-prima o feijão-caupi, a partir de uma revisão sistemática. Foi demonstrado que alimentos que contenham farinhas de feijão-caupi assim como também os sem processamento prévio aumentam significativamente os níveis de vitaminas e minerais, tornando assim o alimento mais nutritivo como um todo. Logo conclui-se que o feijão-caupi carrega consigo uma boa versatilidade em relação a sua aplicabilidade, tornando-o apto para incorporação nas mais diversas formulações alimentícias.

Palavras-chave: Vigna unguiculata; Valor nutritivo; Formulações alimentícias.

\begin{abstract}
The development and enrichment of food is of great importance not only for industry, but also for improving the nutrition of the population, where new products can be created or existing ones can be optimized. Cowpea is one of the main components of the diet in the Northeast and North regions of Brazil, especially in rural areas, due to its high nutritional value, there is a need to expand the diversification of formulations with this raw material, in order to increase the offer of low-cost products, especially for the low-income population. This work aims to identify the main formulations developed, containing cowpea as raw material, based on a systematic review. Foods containing cowpea flour as well as unprocessed foods have been shown to significantly increase levels of vitamins and minerals, thus making the food more nutritious as a whole. Soon it is concluded that the cowpea carries with it a good versatility in relation to its applicability, making it suitable for incorporation in the most diverse food formulations.
\end{abstract}

Keywords: Vigna unguiculata; Nutritional value; Food formulations. 


\section{Resumen}

El desarrollo y enriquecimiento de los alimentos es de gran importancia no solo para la industria, sino también para mejorar la nutrición de la población, donde se pueden crear nuevos productos o optimizar los existentes. El caupí es uno de los principales componentes de la dieta en las regiones Nordeste y Norte de Brasil, especialmente en las zonas rurales, debido a su alto valor nutricional, existe la necesidad de ampliar la diversificación de formulaciones con esta materia prima, con el fin de incrementar la oferta de productos de bajo costo, especialmente para la población de bajos ingresos. Este trabajo tiene como objetivo identificar las principales formulaciones desarrolladas, que contienen caupí como materia prima, a partir de una revisión sistemática. Se ha demostrado que los alimentos que contienen harina de caupí, así como los alimentos no procesados, aumentan significativamente los niveles de vitaminas y minerales, lo que los hace más nutritivos en su conjunto. Luego se concluye que el caupí conlleva una buena versatilidad en relación a su aplicabilidad, lo que lo hace apto para su incorporación en las más diversas formulaciones alimentarias.

Palabras clave: Vigna unguiculata; Valor nutricional; Formulaciones alimentarias.

\section{Introdução}

O feijão-caupi inicialmente foi classificado nos gêneros Phaseolus e Dolichos, até ser classificado no gênero Vigna, o qual foi estabelecido em 1894. O feijão-caupi é uma cultura de origem africana, introduzida no Brasil na segunda metade do século XVI pelos colonizadores portugueses no Estado da Bahia. Existem relatos de que em 1568 já havia a indicação da existência de muitos feijões no Brasil e que em 1587 uma grande variedade de feijões e favas era cultivada na Bahia (Cavalcante, 2018; Filho et al., 2018; Souza, 2018; LPWG, 2017; Rahman, 2015; Wilk \& Barbosa, 2012).

Constitui-se em um dos principais componentes da dieta alimentar nas regiões Nordeste e Norte do Brasil, especialmente na zona rural. Somente as cultivares de feijão-caupi geradas pela Embrapa Meio-Norte, em parceria com outras instituições do sistema cooperativo de pesquisa, ocupam $30 \%$ da área total cultivada com essa leguminosa no país (1.451.578 ha), gerando milhares de empregos diretos e renda (Filho et al., 2018; Souza, 2018; Ribeiro et al., 2013; Kigel \& Rosental, 2015).

Constata-se que o cultivo do feijão está se expandindo para a região dos cerrados, das regiões Norte, Nordeste e CentroOeste, onde é incorporado aos arranjos produtivos como safrinha após as culturas da soja e do arroz, e, em alguns locais, como cultura principal. Na região dos cerrados, principalmente quando é cultivado em forma de safrinha, o feijão-caupi tem um custo muito competitivo, fator que tem feito aumentar o interesse dos produtores pela cultura. A oferta de um produto padronizado, de alta qualidade, em quantidade e com regularidade, vem despertando o interesse de agroindustriais de outras regiões e está contribuindo para a abertura de novos mercados para a cultura (Filho et al., 2018; Souza, 2018 Freire-Filho et al., 2005; Okon, 2013).

De acordo com (SOUZA, 2018, 92p.), recentemente, as técnicas de melhoramento genético, buscando características desejáveis em uma cultivar ideal, originam diversos materiais que compõem os bancos de germoplasma do feijão-caupi. Dentre eles, a cultivar BRS Aracê se caracteriza pela adaptação ao bioma Caatinga sendo indicada para produtores da região Nordeste. Esta cultivar pertence ao grupo comercial cores, subclasse verde e contém alto teor de ferro e zinco no grão, sendo tratada como biofortificada (Monteiro-Araújo et al., 2018; Faostat, 2017; Silva et al., 2016).

Diante disso, o objetivo do presente estudo é identificar quais as principais formulações desenvolvidas, contendo como matéria-prima o feijão-caupi, a partir de uma revisão sistemática.

\section{Metodologia}

O presente trabalho é uma revisão sistemática fundamentada na análise de artigos científicos publicados nos últimos cinco anos qua utilizaram o feijão-caupi no desenvolvimento de produtos, obtidos nas bases de dados Scientific Electronic Library Online (Scielo), PUBMED/MEDLINE e ScienceDirect. Utilizando como descritores as palavras: Vigna unguiculata; Produtos de panificação, Compostos bioativos. Além disso, foram incluídas dissertações e sítios eletrônicos como o da Embrapa (Pereira et al.,2018). 
Foram incluídos estudos publicados em língua inglesa e portuguesa, que apresentaram correspondência com a temática proposta, o que foi verificado a partir da leitura do resumo dos estudos e aqueles que apresentaram um desenvolvimento de um produto alimentício contendo na sua formulação farinha de feijão-caupi. Foram excluídos artigos duplicados, cartas, editoriais, inadequação ao questionamento proposto, artigos indisponíveis.

Após a busca dos artigos na base de dados citadas, utilizando a faixa temporal delimitada juntamente com os descritores, selecionados foram encontrados inicialmente cerca de 500 artigos, após a aplicação dos critérios de inclusão e exclusão restaram 7 artigos e estes foram direcionados para a discussão.

\section{Resultados e Discussões}

O Quadro 1 mostra o delineamento metodológico realizado para filtrar os artigos que foram utilizados no estudo.

Quadro 1. Artigos encontrados inicialmente na busca com base nos descritores.

Pubmed $(\mathrm{n}=152) ;$ Science Direct $(\mathrm{n}=210) ;$ Scielo $(\mathrm{n}=148)$
Excluídos com base nos títulos:
Pubmed ( $\mathrm{n}=98) ;$ Science Direct $(\mathrm{n}=122) ;$ Scielo $(\mathrm{n}=140)$
Resumos selecionados para avaliação:
Pubmed $(\mathrm{n}=45) ;$ Science Direct $(\mathrm{n}=42) ;$ Scielo $(\mathrm{n}=100)$
Excluídos com base nos resumos e da leitura na íntegra:
Pubmed ( $\mathrm{n}=4) ;$ Science Direct $(\mathrm{n}=2) ;$ Scielo $(\mathrm{n}=4)$
Corpus final:
Pubmed $(\mathrm{n}=4) ;$ Science Direct $(\mathrm{n}=2) ;$ Scielo $(\mathrm{n}=3)$

Fonte: Dados da pesquisa (2021).

A partir da pesquisa realizada nas diversas bases de dados, como pode-se observar no Quadro 1, obteve-se um total de 510 artigos utilizando os descritores citados na metodologia, sendo que 360 foram excluídos com base nos títulos que não se enquadraram na pesquisa. 187 resumos foram selecionados para avaliação de seu conteúdo, sendo 10 artigos excluídos com base nos resumos e no texto na íntegra, resultando como corpus final um total de 9 artigos que se enquadravam na proposta apresentada pelo trabalho.

De acordo com a pesquisa realizada pode-se observar a distribuição de artigos por base de dados, no qual ficou demonstrado que a metade dos trabalhos selecionados com os critério de avaliação encontraram-se nas bases de dados Pubmed, seguidos da Scielo, já base de dados ScienceDirect, apresentou um menor volume de trabalhos referentes ao tema.

Após a avaliação dos artigos nas bases de dados selecionadas, aplicando os critérios de inclusão e exclusão, e confrontando as metodologias com os resultados, a partir da identificação de alimentos produzidos contendo farinhas de feijãicaupi, obteve-se 7 artigos que estão descritos na tabela abaixo (Tabela 1). 
Tabela 1. Artigos selecionados na faixa temporal de 2010-2020.

\begin{tabular}{|c|c|c|c|c|}
\hline $\mathbf{A}$ & Artigo & Autores & Ano & Revista \\
\hline A1 & $\begin{array}{c}\text { Pão de queijo enriquecido com } \\
\text { feijão-caupi } \\
\text { Biofortificado e suas } \\
\text { características sensoriais, } \\
\text { nutritivas e funcionais. }\end{array}$ & Cavalcante, et al. & 2016 & Ciênc. Agrotec. \\
\hline A2 & $\begin{array}{l}\text { Vegetal burgers ofcashew fiber } \\
\text { andncowpea: } \\
\text { formulation,characterization } \\
\text { andstability during frozen storage. }\end{array}$ & Lima, J. R. et al. & 2018 & $\begin{array}{c}\text { Ciência } \\
\text { Agronômica }\end{array}$ \\
\hline A3 & $\begin{array}{c}\text { Propriedades tecnológicas, } \\
\text { nutricionais e sensoriais de } \\
\text { biscoito tipo cookie sem glúten, } \\
\text { desenvolvido com arroz de } \\
\text { diferentes teores de amilose e } \\
\text { feijão caupi }\end{array}$ & Souza, E. J. D. et al. & 2018 & $\begin{array}{l}\text { Repositório } \\
\text { UFPEL }\end{array}$ \\
\hline A4 & $\begin{array}{l}\text { Development of a nugget based } \\
\text { on the acerola residue (Malpighia } \\
\text { emarginata D.C) and cowpea } \\
\text { (Vigna unguiculata L.) }\end{array}$ & Abreu, B. B. et al. & 2020 & $\begin{array}{l}\text { Brazilian journal } \\
\text { of Development }\end{array}$ \\
\hline A5 & $\begin{array}{l}\text { Mineral profile and } \\
\text { characterisation of cookies made } \\
\text { from legume green grain flour }\end{array}$ & Maia, L. C. et al. & 2020 & $\begin{array}{c}\text { Food Science } \\
\text { and Technology }\end{array}$ \\
\hline A6 & $\begin{array}{l}\text { Pão de queijo enriquecido com } \\
\text { feijão-caupi biofortificado e suas } \\
\text { características sensoriais, } \\
\text { nutritivas e funcionais }\end{array}$ & Cavalcante, R. B. M. & 2018 & $\begin{array}{l}\text { Repositório } \\
\text { UFPI }\end{array}$ \\
\hline A7 & $\begin{array}{c}\text { Composição centesimal de } \\
\text { cookies a base de farinhas } \\
\text { variadas }\end{array}$ & $\begin{array}{c}\text { Landim, L. A. S. R. } \\
\text { et al. }\end{array}$ & 2019 & $\begin{array}{l}\text { Anais do V } \\
\text { CONAC }\end{array}$ \\
\hline
\end{tabular}

*A = artigo. Fonte: Dados da pesquisa (2021).

O estudo A1 verificou que os alimentos à base de grãos integrais, incluindo o pão, desempenham um papel importante na saúde humana e no bem-estar. Demonstrou-se que o consumo regular de produtos integrais pode contribuir para reduzir o risco de doenças cardiovasculares (DCV), diabetes mellitus tipo 2 e certos tipos de câncer, bem como várias patologias gastrointestinais. As propriedades saudáveis dos grãos integrais estão ligadas à presença de compostos bioativos, como fibra alimentar e compostos fenólicos. Além do acréscimo de fenólicos totais, a inclusão de farinhas integrais para obtenção de pães melhora o conteúdo de proteínas, vitaminas e minerais, como também a qualidade tecnológica do produto (Cavalcante, et al., 2016). Portanto, faz-se necessário um estudo clínico com o produto formulado para poder correlacionar com os benéficos citados pelo autor ao longo do trabalho.

Tem-se demonstrado que o amido proveniente do feijão-caupi melhora a qualidade tecnológica de produtos de panificação quando comparado com o amido de fontes convencionais (mandioca, milho, batata e arroz). Com o amido dessa leguminosa, os batidos apresentam menores valores de gravidade específica e os produtos maiores valores de volume específico, cor mais clara, menor redução da umidade durante o período de estocagem e melhores valores nos atributos de textura durante a vida de prateleira ocasionando maiores escores na avaliação sensorial, especialmente para os atributos do miolo e textura (Souza et al., 2018; Filho et al., 2018; Abreu et al., 2020). Características que fazem desse vegetal uma matéria-prima em potencial para sua aplicabilidade em produtos já existentes, no intuito de agregar valor nutritivo em sua composição.

O estudo que avaliou a funcionalidade do feijão-caupi e o impacto da ingestão de biscoitos à base da farinha da cultivar BRS Xiquexique (pacote de 30g / 3 vezes por semana, durante 60 dias) por pré-escolares suscetíveis a anemia ferropriva. Os resultados demonstraram que a anemia, antes da intervenção, a prevalência era de 12,2\% e após a intervenção 1,4. Logo, 
constatou-se a eficácia do biscoito acrescido de feijão-caupi visto que o mesmo obteve ótima aceitação pelas crianças (94,3\%) (Landim et al., 2019; Moreira-Araújo, et al., 2018; Pereira et al., 2016; Cavalcante et al., 2016). Corroborando com a viabilidade de substituição de farinhas brancas pelas de feijão-caupi e o incremento nas refeições de indivíduos vulneráveis a carências nutricionais.

O A2 mostrou que o feijão-caupi é abundante no Brasil e pode ser usado para aumentar a palatabilidade e o valor da proteína de hambúrgueres feitos de fibra de caju. Feijão-caupi (Vigna unguiculata L. Walp) é uma leguminosa anual comumente conhecido como ervilha-do-sul, ervilha-preta, alubia, caupi, fita ou frijole (Lima, et al., 2018). Em relação ao artigo, o objetivo deste trabalho foi obter um hambúrguer de vegetais da produção de suco de caju resíduo (fibra) misturado com feijão-caupi (fonte de proteína) e avaliar sua composição, aceitação sensorial e estabilidade durante 180 dias de armazenamento congelado $\left(-18^{\circ} \mathrm{C}\right)$.

Desta forma, o hambúrguer de vegetais assim desenvolvido é considerado um produto de baixo teor de gordura e baixo consumo de energia e mostra boa aceitação sensorial. Apesar do ligeiro mudanças físico-químicas durante o armazenamento, é considerado microbiologicamente seguro e sensorialmente aceitável para consumo ao longo dos 180 dias de armazenamento congelado (Lima, et al., 2018). Na qual o torna uma excelente alternativa nutritiva em relação aos já existentes no mercado.

O estudo A3 avaliou os efeitos de farinhas mistas de arroz, com diferentes teores de amilose, e feijão-caupi sobre propriedades tecnológicas, nutricionais e sensoriais de biscoitos tipo cookie e conclui-se que a farinha de feijão apresentou maiores teores de proteína, diferindo significativamente das farinhas de arroz, fato este estando de acordo com Filho et al. (2018), que encontraram de 21,5 a 26,8\% de proteína em diferentes cultivares de feijão-caupi.

O artigo mostrou que o feijão apresenta em sua constituição todos os aminoácidos essenciais, sendo rico em lisina, mas limitante em aminoácidos sulfurados - metionina e cisteína. Em avalição de aminoácidos em leguminosas, verificaram a presença de 410 mg.g-1 N de lisina e 120 mg.g-1 N de metionina em feijão-caupi. Já em avaliação de vários cereais, relataram encontrar em arroz 3,3 (g.g-1 de proteína) de lisina e 2,6 (g.g-1 de proteína) de metionina. Desse modo, o feijão pode ser considerado complementar ao arroz, que é deficiente em lisina e relativamente rico em aminoácidos sulfurados (Abreu et al., 2020; Maia et al., 2020; Souza et al., 2018).

O A4 consiste em um estudo de caráter experimental, realizado na Universidade Federal do Piauí-UFPI, Departamento de Nutrição, no Laboratório de Desenvolvimento de Produtos e Análise Sensorial de Alimentos-LASA, no período de fevereiro à junho de 2018. E objetivou elaborar um "nugget” utilizando o resíduo de acerola e o feijão-caupi, tendo em vista o seu valor nutritivo e características funcionais. Nos resultados do teste discriminativo Pareado de Preferência, foi possível observar que a preferência pelo "nugget" com resíduo de acerola e feijão-caupi foi similar à preferência pelo produto padrão. Dentre os assessores, 53 (49\%), preferiram o produto padrão, enquanto 56 assessores (51\%), preferiram o "nugget" desenvolvido. Estatisticamente, o teste do F2 (qui-quadrado) não mostrou diferença significativa entre os produtos, sendo $\mathrm{p}=0,777$ (Abreu, et al., 2020).

Na Análise Descritiva Quantitativa-ADQ de 7 pontos, foi atribuído ao produto as seguintes descrições para aparência, aroma, sabor e textura, respectivamente: "nugget" com cobertura e textura crocante e macia, aroma e sabor de frango. Não foi observado pelos assessores sensoriais gosto residual da fibra de acerola ou do feijão-caupi. As médias das notas obtidas foram satisfatórias, sendo todas maiores que 4. No atributo sabor, foi atribuída nota acima de 5, com desvio padrão baixo. Este atributo é considerado de grande importância, pois tende a refletir a opinião geral do assessor. Na aparência também se obteve uma ótima avaliação, com nota acima de 5(Abreu, et al., 2020).

O artigo A5 foi comprovado que quando se utiliza farinhas de feijão-caupi em formulações, as mesmas se apresentam como uma ótima alternativa na formulação de biscoitos, uma vez que os resultados indicam que os biscoitos preparados são uma 
boa fonte de proteínas, fibras, carboidratos e minerais essenciais (principalmente K, Ca e P), melhorando a qualidade nutricional do produto final (Maia, et al., 2020).

Em relação ao artigo A6 foi analisado às formulações de pão de queijo que utilizavam farinha de feijão-caupi, foram relatados nos trabalhos pesquisados que os níveis de minerais, cobre, ferro, fósforo, magnésio, manganês e zinco foram significativamente aumentadas, por outro lado, as concentraçõe de sódio e cálcio foram diminuídas (Cavalcante, 2018; Cavalcante, et al., 2016).

No estudo A7 resultou que as formulações de cookies com proporções variadas quanto a presença de farinha de feijãocaupi, farinha de trigo e farinha de soja na composição apresentaram características promissoras em relação aos teores de umidade, cinzas, minerais, proteínas, lipídios e fibras. (Landim, et al., 2019). Podendo assim, ser utilizada nas mais diversas formulações de massas e biscoitos e influenciar positivamente em suas características físico-química.

\section{Conclusões}

Este estudo permitiu concluir que o feijão-caupi possui atributos desejáveis, como alto conteúdo protéico, de fibras alimentares e de minerais e antioxidante, tais como: ferro e zinco e constatou-se baixo teor de lipídios. Do mesmo modo, foi demonstrado a alta versatilidade em relação a farinha de feijão-caupi, sendo utilizada nas mais diversas formulações alimentícias, como pães, cookies, hambúrguer e biscoitos.

Diante dos resultados com os produtos desenvolvidos a partir da farinha de feijão-caupi, conclui-se que ela tem uma relevância significativa quando aplicada nos diversos alimentos, agregando valor nutricional. Com isso, mostra que essa leguminosa tem um elevado potencial no mercado internacional como matéria-prima para novos estudos e pesquisas de novas formulações com de intervenção.

\section{Referências}

Abreu, B. B., Ferraz, L. R. L., Cavalcante, R. B. M., Campos, C. M. F., Gonçalves, M. F. B., Oliveira, E. L. C., Brandão, A. C. A. S., \& Araújo, R. S. R M. (2020). Desenvolvimento de um "nugget” à base do resíduo da acerola (Malpighia emarginata D.C) e feijão-caupi (Vigna unguiculata L.). Braz. Jour. Dev., 6(2), 9446-9453. https://doi.org/10.34117/bjdv6n2-307

Cavalcante, R. B. M. (2018).Pão de queijo enriquecido com feijão-caupi biofortificado e suas características sensoriais, nutritivas e funcionais. 2018. 121f. Tese (Doutorado) - Programa de Pós-Graduação em Alimentos e Nutrição. Universidade Federal do Piauí, Teresina. https://www.google.com/url?sa=t\&rct=j\&q=\&esrc=s\&source=web\&cd=\&cad=rja\&uact=8\&ved=2ahUKEwib95GhsrjwAhWDppUCHfIJCe8QFnoECAUQA A\&url=https\%3A\%2F\%2Frepositorio.ufpi.br\%2Fxmlui\%2Fhandle\%2F123456789\%2F1394\&usg=AOvVaw0f4YdBBjawc0pNUhnJHudN

Cavalcante, R. B. M., Morgano, M. A., Silva, K. J. D., Rocha, M. M., Araújo, M. A. M., \& Moreira-Araújo, R. S. R. (2016). Cheese bread enriched with biofortified cowpea flour. Ciência e Agrotecnologia, 40(1), 97-103. https://doi.org/10.1590/S1413-70542016000100009

Faostat, F. Food And Agriculture Organization Of The United Nations. FAO. Crops: cowpeas, dry (2017). http://faostat3.fao.org/browse/Q/QC/E

Freire-Filho, F. R., Lima, J. A. A., \& Ribeiro, V. Q. Feijão-caupi: avanços tecnológicos. Brasília: Embrapa Informação Tecnológica, 2005. 519p.

Kigel, J., Rosental, L., \& Fait, A. Seed Phisiology and Germination of Grain Legume. In: De Ron A. (eds) Grain Legume. Handbook of Plant Breeding. New York: Springer, 2015. p.327-363.

Landim, L. A. S. R., Silva, K. J. D., Hashimoto, J. M., \& Rocha, M. M. (2019). Composição centesimal de cookies a base de farinhas variadas. Anais do V CONAC (Embrapa). 1(1), 1-4. https://www.embrapa.br/busca-de-publicacoes/-/publicacao/1117885/composicao-centesimal-de-cookies-a-base-de-farinhasvariadas

Lima, J. R. et al. Vegetal burgers of cashew fiber and cowpea: formulation, characterization and stability during frozen storage. Rev. Ciênc. Agron., 49(4), 708714.

LPWG - The Legume Phylogeny Working Group. A new subfamily classification of the Leguminosae based on a taxonomically comprehensive phylogeny. Taxon,66(1),44-77, 2017.https://doi.org/10.12705/661.3

Okon, J. E. Effect of Water stress on some growth aspects of two cultivars of cowpea, Vigna unguiculata (L.) Walp Fabaceae. Bulletin of Environment, Pharmacology and Life Sciences, 2(5),.69-74. http://www.bepls.com/april_2013/12.pdf

Maia, L. C., Nano, R. M. W., Santos, W. P. C., Nascimento, P. V. B. S., Miranda, K. E. S., Oliveira, F. S. (2020). Mineral profile and characterisation of cookies made from legume green grain flour. Food Science and Technology, https://doi.org/10.1590/fst.22020 
Research, Society and Development, v. 10, n. 14, e56101416054, 2021

(CC BY 4.0) | ISSN 2525-3409 | DOI: http://dx.doi.org/10.33448/rsd-v10i14.16054

Pereira, E. J., Carvalho, L. M., Dellamora-Ortiz, G. M., Cardoso, F. S., \& Carvalho, J. L. (2016). Effect of different home-cooking methods on the bioaccessibility of zinc and iron in conventionally bred cowpea (Vigna unguiculata L. Walp) consumed in Brazil. Food \& nutrition research, 60, 29082. https://doi.org/10.3402/fnr.v60.29082

Pereira et al. (2018). Metodologia da pesquisa científica.

Rahman, A. H. M., \& Parvin, M. I. A. Taxonomic Studies on the family Fabaceae (Weeds) at Rajshahi University Campus. Plant,3(3),20-25, 2015.https://doi.org/10.11648/j.plant.20150303.11

Ribeiro, G. S., Ferreira, A. F., Lyra N. C. M.; Mercês S. F. S., Oliveira, C., Alves, E. M., Silva, S. G., \& Carvalho, C. A. L. Aspects of the floral biology and pollen properties of Vigna unguiculata L. Walp (Fabaceae). African Journal of Plant Science, 7(5), 149-154, 2013.https://doi.org/10.5897/AJPS13.1014

Silva, K. J. D., Rocha, M. M., \& Menezes-Junior, J. A. Socioeconomia. In: Bastos, E. A. A cultura do feijão-caupi no Brasil. Teresina: Embrapa Meio-norte, 2016. 6-12.

Souza, A. C. M. Análise transcriptômica das vias metabólicas do inositol e de oligossacarídeos da família rafinose, em feijão-caupi sob estresse abiótico e biótico. 2019. Tese (Doutorado em Ciências Biológicas) - Universidade Federal de Pernambuco, Recife, 2019. https://repositorio.ufpe.br/bitstream/123456789/35338/1/TESE\%20Amanda\%20Cordeiro\%20de\%20Melo\%20Souza.pdf

Souza, E. J. D. (2018). Propriedades tecnológicas, nutricionais e sensoriais de biscoito tipo cookie sem glúten, desenvolvido com arroz de diferentes teores de amilose e feijão-caupi. 2018. 92f. Dissertação (Mestrado) - Programa de Pós-Graduação em Ciência e Tecnologia de Alimentos. Universidade Federal de Pelotas, Pelotas. http://guaiaca.ufpel.edu.br/handle/prefix/4093

Wilk, R., \& Barbosa, L. Rice and beans: a unique dish in a hundred places. Berg, 2012. 282p. 